\title{
An Ultrawideband Signal Design with Power Spectral Density Constraints
}

\author{
(Invited Paper) \\ Terry P. Lewis ${ }^{* \dagger}$, Robert A. Scholtz ${ }^{\dagger}$ \\ ${ }^{*}$ Raytheon \\ Networked Centric Systems, Fullerton, California 92833-2200 \\ Email: tplewis@ raytheon.com \\ †Electrical Engineering Systems \\ University of Southern California, Los Angeles Ca 90089-2565 \\ Email: terry@usc.edu, scholtz@usc.edu
}

\begin{abstract}
An UWB signal was designed and generated using a zero order digital to analog converter. An efficiency measure of filling the FCC spectral mask is developed and optimized for various choices of digital-to-analog converter parameters and waveform duration. The proposed optimization technique takes into account filtering effects in the digital-to-analog converter as well as a measured antenna transfer function characteristic.
\end{abstract}

\section{An Introduction to The FCC Mask Constraint}

The FCC has imposed a mask $M(f)$ (see Figure 1) to bound the power spectral density $S_{\mathrm{EIRP}}(f)$ of the equivalent isotropic radiated power (EIRP) of a UWB communication signal [1]. The detailed shape of $M(f)$ depends on the particular UWB system application. For many modulation formats, the power spectral density (PSD) of a modulated train $x(u, t)$ of pulses $p(t)$ factors as [2], [3]

$$
S_{x}(f)=S_{m}(f)|P(f)|^{2},
$$

where $S_{m}(f)$ is is a function only to the modulation process, and $P(f)$ is the Fourier transform of the UWB pulse shape $p(t)$, i.e.,

$$
P(f)=\int_{0}^{\tau} p(t) \exp (-j 2 \pi f t) d t .
$$

The transformation from the modulated pulse train in the transmitter to the radiated electromagnetic far field on which the EIRP mask is imposed, is a linear transformation which we represent by system function $H_{\mathrm{eq}}(f)$. Hence the power spectral density $S_{\mathrm{EIRP}}(f)$ which is bounded by the mask $M(f)$ is of the form

$$
S_{\mathrm{EIRP}}(f)=S_{x}(f)\left|H_{\mathrm{eq}}(f)\right|^{2}=S_{m}(f)|P(f)|^{2}\left|H_{\mathrm{eq}}(f)\right|^{2} .
$$

We assume further PSD of the modulation process is approximately a constant for all $f$ of interest, and without loss of generality we assume that $S_{\mathrm{EIRP}}(f) \approx a_{1}|P(f)|^{2}\left|H_{\mathrm{eq}}(f)\right|^{2}$. The constant $a_{\mathrm{c}}$ includes factors that convert the units of $\left|P(f) H_{\text {eq }}(f)\right|^{2}$ (typically volts ${ }^{2} /$ Hertz) to the units of $M(f)$ (typically Watts/megahertz), in addition to scaling by the constant spectral density of the modulation. Hence the FCC requirement is that

$$
a_{\mathrm{c}}|P(f)|^{2}\left|H_{\mathrm{eq}}(f)\right|^{2} \lesssim M(f)
$$

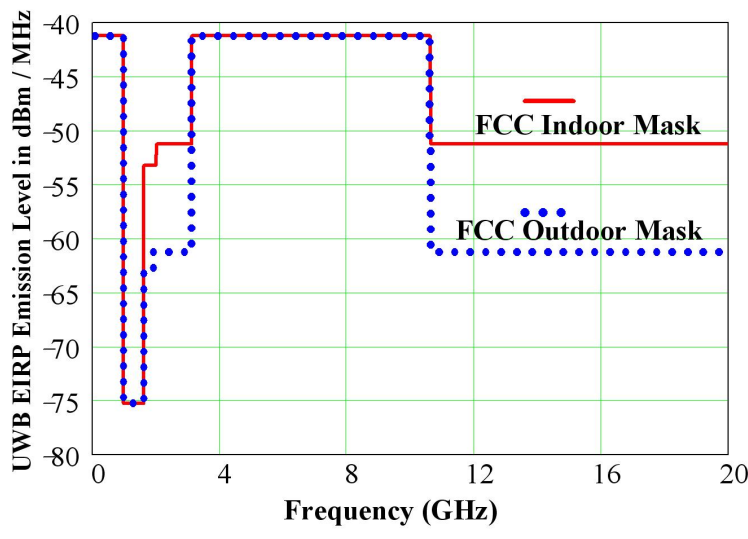

Fig. 1. FCC Outdoor and Indoor PSD Constraints

for all $f$ in the measurement range $\left(F_{\min }, F_{\max }\right)$.

\section{MASK-FILLING EFFICIENCY}

The mask-filling efficiency $\eta$ of a particular signal design can be defined as the ratio of the power contained in the resulting FCC-compliant $\operatorname{PSD} S_{\mathrm{EIRP}}(f)$ to the total power that could be contained under the mask $M(f)$, both being evaluated in the measurement range. Hence we define

$$
\eta \triangleq \frac{a_{\mathrm{c}} \int_{F_{\min }}^{F_{\max }}|P(f)|^{2}\left|H_{\mathrm{eq}}(f)\right|^{2} d f}{\int_{F_{\min }}^{F_{\max }} M(f) d f} .
$$

This mask-filling efficiency measure for a pulse waveform $p(t)$, given the system function $H_{\mathrm{eq}}(f)$, also embodies the effects of transmitter, antenna, and propagation on signals. Let $\mathcal{P}_{\mathrm{PG}}$ be the set of possible pulse waveforms $p_{n}(t)$ that can be implemented in the pulse generator. Here the subscript $n$ simply indexes the elements in $\mathcal{P}_{\mathrm{PG}}$. Furthermore for each waveform in $\mathcal{P}_{\mathrm{PG}}$, a scale factor $A_{n}$ can be chosen so that

$$
p(t)=A_{n} p_{n}(t)
$$

and the FCC mask bound in (4) is satisfied by $p(t)$ for all $f \in\left(F_{\min }, F_{\max }\right)$, and is achieved with equality for at least 
one value of $f$ in this measurement range. This constant $A_{n}$ can always be implemented in the transmitter as an all-band amplification/attenuation.

\section{Pulse Waveform Selection Procedure}

1) For each possible waveform $p_{n}(t) \in \mathcal{P}^{*}$, find the constant $A_{n}$ so that (4) is satisfied for all $f$ and achieved for some $f$ in the measurement range. This computation in $\mathrm{dB}$ form is equivalent to evaluating

$$
\begin{aligned}
\left(a_{\mathrm{c}} A_{n}^{2}\right)_{\mathrm{dB}} & =\min _{f \in\left(F_{\min }, F_{\mathrm{max}}\right)}\left[(M(f))_{\mathrm{dB}}\right. \\
& \left.-\left.\left(\mid H_{\mathrm{eq}}(f)\right)\right|_{\mathrm{dB}} ^{2}-\left(\left|P_{n}(f)\right|^{2}\right)_{\mathrm{dB}}\right] .
\end{aligned}
$$

2) For each possible waveform $p_{n}(t) \in \mathcal{P}^{*}$, calculate the mask filling efficiency $\eta_{n}$ of the waveform $p_{n}(t)$ by evaluating

$$
\eta_{n} \triangleq \frac{a_{\mathrm{c}} A_{n}^{2} \int_{F_{\min }}^{F_{\max }}\left|P_{n}(f)\right|^{2}\left|H_{\mathrm{eq}}(f)\right|^{2} d f}{\int_{F_{\min }}^{F_{\max }} M(f) d f} .
$$

3) Find the waveform $p_{n_{\text {opt }}}(t)$ with the highest efficiency $\eta_{\max }$, i.e.,

$$
\eta_{\max }=\max _{1 \leq n \leq N} \eta_{n}
$$

and

$$
n_{\mathrm{opt}}=\underset{1 \leq n \leq N}{\arg \max } \eta_{n} .
$$

This procedure describes an exhaustive search, and hence is applicable to situations in which the set $\mathcal{P}^{*}$ is finite.

\section{COMMENTS ON PRIOR WORK}

If there were no constraints on the pulse shape $p(t)$, then it may be possible to determine a waveform $p(t)$ with $100 \%$ mask-filling efficiency by solving

$$
|P(f)|^{2}=\frac{M(f)}{a_{\mathrm{c}}\left|H_{\mathrm{eq}}(f)\right|^{2}}
$$

for $P(f)$. This assumes that the right hand side of (9) can represent the squared magnitude of a finite energy waveform. Factorizations of this type that recover a pulse shape $p(t)$ with time support $[0, \infty)$ can be carried out provided that $M(f) /\left|H_{\mathrm{eq}}(f)\right|^{2}$ satisfies Paley Wiener conditions. Then, the optimal pulse waveform $p(t)$ can be recovered by a Fourier transform inversion of $P(f)$.

Slepian et al. studied methods to optimize the concentration of energy in a band of frequencies for functions with finite time support, using prolate spheroidal wave functions for continuous time [4] and discrete time [5] systems. (see also [6], [7], [8].) Slepian's problem formulation differs from our approach in the following respects:

1) Here $H_{\text {eq }}(f)$ is not an ideal all-pass filter. Any application of this prior work to the problem stated here must assume $H_{\mathrm{eq}}(f)=1$.

2) Here we are constrained by a PSD mask, while the prior work only maximizes in-band power, without concern for the shape of the in-band power density.

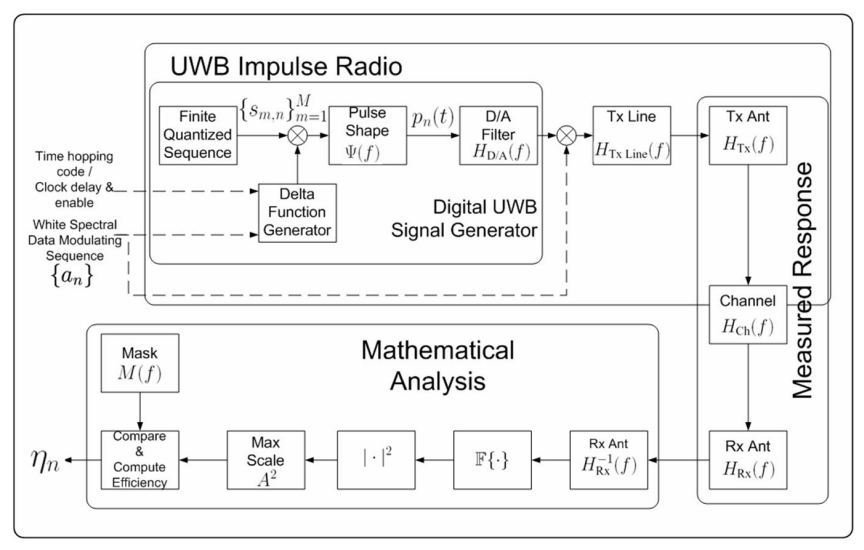

Fig. 2. System Diagram Model

3) The allowed waveform set in the prior work is different from $\mathcal{P}^{*}$.

The use of prolate spheroidal functions as UWB signals has been suggested [9].

\section{A UWB Pulse Generator}

Viable UWB pulse generators with the ability to produce complex pulse waveforms are difficult to construct. Here we assume that the UWB pulse generator (see Figure 2) is a zero-order digital-to-analog converter (DAC) which produces a pulse signal of the form

$$
p_{n}(t)=\sum_{m=0}^{M-1} s_{m, n} \psi\left(t-m T_{\mathrm{s}}\right)
$$

from an input $M$-tuple $s_{m, n}, m=0, \ldots, M-1$. The ideal elementary waveform $\psi(t)$ is a unit-energy rectangular pulse,

$$
\psi(t)= \begin{cases}1 / \sqrt{T_{\mathrm{s}}}, & \text { if } 0<t \leq T_{\mathrm{s}} \\ 0, & \text { otherwise, }\end{cases}
$$

that is reproduced at a rate $T_{\mathrm{s}}^{-1}$ and scaled to produce a staircase waveform with time support $M T_{\mathrm{s}}$. Further filtering of this signal form (11) within the DAC are included in the model of $H_{\mathrm{eq}}(f)$. The scaling coefficients $s_{m, n}$ are chosen from a set $\mathcal{Q}$ of coefficients that are uniformly spaced. Hence, the range $R$ of this uniform quantization is given by

$$
R=(|\mathcal{Q}|-1) \Delta_{\text {step }},
$$

where $\Delta_{\text {step }}$ is the magnitude of the difference between any two adjacent elements of $\mathcal{Q}$. The number $N$ of distinct waveforms of duration $M T_{\mathrm{s}}$ that the DAC can produce is

$$
N \triangleq|\mathcal{Q}|^{M} .
$$

In terms of the general problem construct, $N=\left|\mathcal{P}^{*}\right|$.

The coefficient $M$-tuple $s_{m, n}, m=0, \ldots, M-1$, used by the DAC to generate $p_{n}(t)$ can be generated systematically for a uniform symmetric quantization by representing $n$ as a base 
$|\mathcal{Q}|$ number and using the elements in this representation as the coefficient $M$-tuple. That is,

$$
s_{m, n}=\left\{\left[\frac{n}{|\mathcal{Q}|^{m}}\right] \bmod |\mathcal{Q}|\right\} \Delta_{\text {step }}-\frac{R}{2},
$$

where $0 \leq m<M, 0 \leq n<N$ and $[x]$ represents the integer part of $x$. Choosing $\Delta_{\text {step }}=2$ insures for simplicity that the coefficients $s_{m, n}$ are integers for both even and odd choices of $|\mathcal{Q}|$. We can view the coefficients $s_{m, n}$ as entries in an $M \times N$ matrix $\mathbf{S}_{M \times N}$ matrix whose row and column indices range over $0 \leq m<M, 0 \leq n<N$ respectively.

The DAC performs the mapping $\mathbf{s}_{n} \rightarrow p_{n}(t)$ according to (10) where $\mathbf{s}_{n}$ is the $n^{\text {th }}$ column of $\mathbf{S}_{M \times N}$. The Fourier transform of $p_{n}(t)$ in (10), which is required to carry out the waveform selection procedure, is

$$
P_{n}(f)=\mathbb{F}\left\{p_{n}(t)\right\}=\Psi(f) \sum_{m=0}^{M-1} s_{m, n} \exp ^{-j 2 \pi f m T_{\mathrm{s}}},
$$

where $\mathbb{F}\{\psi(t)\}=\Psi(f)$.

\section{The Signal-Path System Function}

To complete the model for optimization, we must determine the system function $H_{\text {eq }}(f)$ of the signal path from the signal generator output $p_{n}(t)$ to the strongest portion of the radiated electric far field $\vec{\rho} E(t)$ at a distance $r$ from the radiating antenna. The power spectral density of the amplitude $E(t)$ of this field is multiplied by $4 \pi r^{2}$ to produce the measured isotropic radiated power spectral density $S_{\mathrm{EIRP}}(f)$. The system function $H_{\mathrm{eq}}(f)$ has several factors that can be identified in Figure 2, each representing the effect of a portion of the signal path from generated pulse $p_{n}(t)$ to the electric field $E(t)$.

Filtering in the DAC: The output of the D/A converter filter is

$$
\mathbb{F}\left\{p_{n}(t) * h_{\mathrm{D} / \mathrm{A}}(t)\right\}=P_{n}(f) H_{\mathrm{D} / \mathrm{A}}(f),
$$

In the sample computations done here, the system function $H_{\mathrm{D} / \mathrm{A}}(f)$ was determined from a measured response of a real UWB digital synthesizer. The step response of the signal generator was measured and it was determined that the signal generator had a $90 \%$ risetime of $\sim 41 \mathrm{ps}$. The shape of the leading edge of the response was found to fit well with the shape of a Gaussian cumulative distribution function. Using the properties of random variables to compute the parameters of a zero mean gaussian density function, the filter's impulse response variance was calculated to be $\sigma_{\mathrm{D} / \mathrm{A}}^{2} \cong 1.25 \mathrm{E}-$ $11 \mathrm{sec}^{2}$, and thus the DAC filter is completely determined by

$$
h_{\mathrm{D} / \mathrm{A}}(t)=\frac{1}{\sqrt{2 \pi \sigma}} e^{\frac{-\left(t-\mu_{t}\right)^{2}}{2 \sigma^{2}}},
$$

where for simplicity, the mean $\mu_{t}=0$. The specified Gaussian density filter has a $3 \mathrm{~dB}$ bandwidth of $10 \mathrm{GHz}$ and the normalized (at DC) transfer function of $h_{\mathrm{D} / \mathrm{A}}(t)$ is

$$
\begin{aligned}
\mathbb{F}\left\{h_{\mathrm{D} / \mathrm{A}}(t)\right\} & =H_{\mathrm{D} / \mathrm{A}}(f) \\
& =1.0 \exp \left[-7.8 \cdot 10^{-23}(2 \pi f)^{2}\right] .
\end{aligned}
$$

Transmission Lines: The transmission line to the antenna exhibits a constant impedance (typically $50 \Omega$ ) across a wide frequency band of operation. We assume in the sample computations done here that the transmission line behaves like an ideal all-pass filter across the measurement band and can be ignored. Distortion of ultra fast pulses in transmission lines has been studied [10] and results have shown that distortions do occur if precautions are not taken.

Transmitting Antenna and Radiation: For most antennas the transfer functions of interest may have to be measured or simulated [11], [12]. Pozar has used analytical models for antennas over wide bandwidths [13]. For the antennas considered, his results indicate that the transfer function from the antenna input to the radiated far field and to the receiving antenna are not flat over the band of interest, and hence antennas can have a significant spectral shaping effect on an UWB waveform. This is true generally because of the wide bandwidths used and the difficulty of constructing simple UWB antennas which are relatively flat across the bandwidth of interest.

Three pairs of antennas were used in our sample computations:

- Transverse Electromagnetic Mode (TEM) Horn antennas - TEM horn antennas were used in the experiment because they exhibit relatively constant gain, good impedance match and linear phase over several decades of frequency. Their performance is very predictable using mathematical models [14], [15], [16]. The features of TEM horns are typically difficult to achieve in simple antennas.

- small TEM Horn antennas - The small TEM horn antennas were designed to have a slightly higher cut on frequency relative to the larger TEM horm antennas. Both types of TEM horns were designed with truncated ground planes for compactness of size, causing somewhat degraded behavior.

- diamond dipole antennas - The diamond dipole antennas are relatively small antennas that were proposed for UWB use but are too large for hand held use as well.

The measured $S_{2-1}$ responses shown in Figure 3, were measured in an anechoic chamber by a calibrated network analyzer. The calibration was performed up to the antenna input terminals and 1601 uniformly spaced data samples were taken from $50 \mathrm{MHz}-20.05 \mathrm{GHz}$. In all cases, pairs of identical antennas were used in the measurement process. A relatively large reflection can be observed around $18 \mathrm{GHz}$. This reflection is caused by the limitations of the SMA connectors and were dampened for the analysis.

Defining $H_{\text {meas }}(f) \triangleq S_{2-1}$, it is possible to decompose this measured function as

$$
H_{\text {meas }}(f)=H_{\mathrm{tx}}(f) H_{\mathrm{ch}}(f) H_{\mathrm{rx}}(f),
$$

where $H_{\mathrm{tx}}(f), H_{\mathrm{ch}}(f)$ and $H_{\mathrm{rx}}(f)$ are the system functions of the transmit antenna, free space environment and the receiving antenna respectively. The last factor $H_{\mathrm{rx}}(f)$ is the transfer function from the electric field in the vicinity of the receiving 


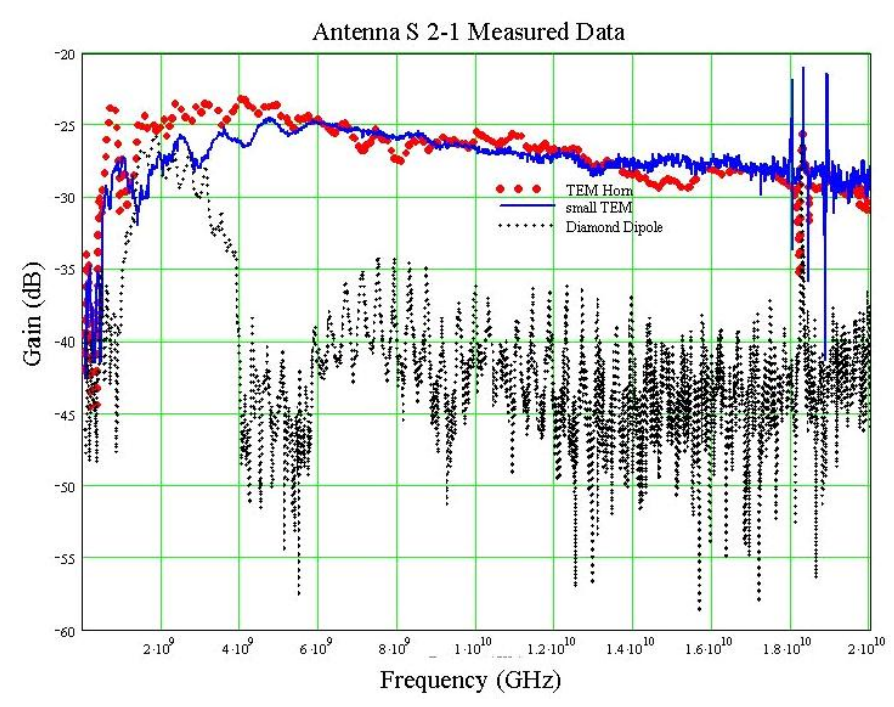

Fig. 3. Antenna S21 plots

antenna to the receiving antenna terminals, and its effect must be removed by calibration or analytically [16], [17].This explains the use of the inverse receive antenna transfer function in Figure 2.In summary,

$$
H_{\mathrm{eq}}(f)=H_{\mathrm{D} / \mathrm{A}}(f) H_{\mathrm{TxLine}}(f) H_{\text {meas }}(f) H_{\mathrm{rx}}^{-1}(f),
$$

and all of these factors must be considered in the modelling process.

\section{Search Space Reduction}

We implemented an exhaustive search for DAC-generated pulse waveforms that have the highest efficiency. For a given set of the parameters $T_{\mathrm{s}}, H_{\mathrm{eq}}, M$ and $N$ and assuming uniform symmetric quantization in the DAC, this involves constructing the coefficient $\mathbf{S}_{M \times N}$, and for each column $\mathbf{s}_{n}$ of the matrix, compute the corresponding $A_{n} p_{n}(t),\left|A_{n} P_{n}(f)\right|^{2}$ and maskfilling efficiency $\eta_{n}$. This chain of mappings from $\mathbf{s}_{n}$ to $\eta_{n}$ is many-to-one, We can construct an equivalence relation of the form

$$
\mathbf{s}_{j} \equiv \mathbf{s}_{k} \quad \Longleftrightarrow \quad\left|A_{j} P_{j}(f)\right|^{2}=\left|A_{k} P_{k}(f)\right|^{2}
$$

that creates equivalence classes of coefficient vectors which produce the same EIRP density and hence the same maskfilling efficiency. In the search for the coefficient vectors $\mathbf{s}_{n}$ that produce waveforms with the highest mask-filling efficiency, the efficiency of only one coefficient vector from each equivalence class needs to be evaluated. Under (21), two coefficient vectors $\mathbf{s}_{j}$ and $\mathbf{s}_{k}$ will be equivalent if either

$$
\mathbf{s}_{j}=c \mathbf{s}_{k}
$$

for some (positive or negative) constant $c$, or

$$
s_{j, m}=s_{k, M-1-m} \quad \text { for all } m \in\{0,1, \ldots, M-1\} .
$$

The first condition (22) involves scaling which is compensated in the search algorithm by adjusting the weight $A_{n}$ in step 1 of the selection procedure. The second condition (23) is
PSD of the best 3 waveforms for large TEM horn

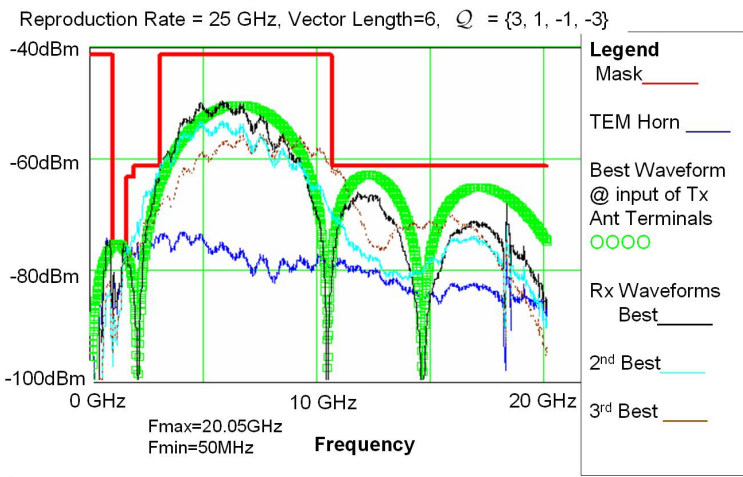

Fig. 4. Best result for TEM Horn $m=1,2, \ldots, 7$

based on the fact that reversing the order of the elements in a coefficient vector time-reverses the real pulse waveform, thereby conjugating its Fourier transform but leaving the EIRP density unchanged. As an example, consider set of 2-tuples represented by the coefficient matrix S. Here $M=2,|\mathcal{Q}|=$ $2^{M}, \mathcal{Q}=\{-3,-1,1,3\}$ and $N=16$ (see (13)), where the set $\mathcal{Q}$ was formed from (see (14)). Then the coefficient matrix $\mathbf{S}$ is given by

$$
\mathbf{S}=\left[\begin{array}{rrrrrrrr}
-3 & -1 & 1 & 3 & -3 & -1 & 1 & 3 \\
-3 & -3 & -3 & -3 & -1 & -1 & -1 & -1 \\
-3 & -1 & 1 & 3 & -3 & -1 & 1 & 3 \\
1 & 1 & 1 & 1 & 3 & 3 & 3 & 3
\end{array}\right] .
$$

Eliminating all but one of each set of equivalent column vectors from the coefficient matrix produces a matrix $\tilde{\mathbf{S}}$ whose columns are equivalence class representatives.

$$
\tilde{\mathbf{S}}=\left[\begin{array}{rrrr}
-1 & -1 & 1 & 1 \\
-1 & -3 & -3 & -1
\end{array}\right]
$$

This matrix is not unique, the only requirement being that one representative from each equivalence class should appear as a column in $\tilde{\mathbf{S}}$. Applying the pulse waveform selection procedure to the set pulse waveforms generated by the columns of $\tilde{\mathbf{S}}$ will find the equivalence class representative of the set of columns that produce waveforms with the highest mask-filling efficiency.

\section{Results AND CONCLUSIONS}

Results of searches for various sets of parameters are typical of the results shown in Figures 4-6 where 4 shows the three waveforms with the highest efficiency. Figure 5 indicates that the optimal waveform $p_{\text {opt }}(t) \in \mathcal{P}^{*}$ are rare events. This was typical of all antennas investigated. Figure 6 summarizes in 3D space, the search results of the Large TEM Horn. Different surface plots were observed for the small TEM horn and diamond dipole antennas, using the same search parameters as in the large TEM horn. It was observed that we get different optimal designs for different parameters e.g., antennas and in all cases low levels of efficiency were achieved. This 


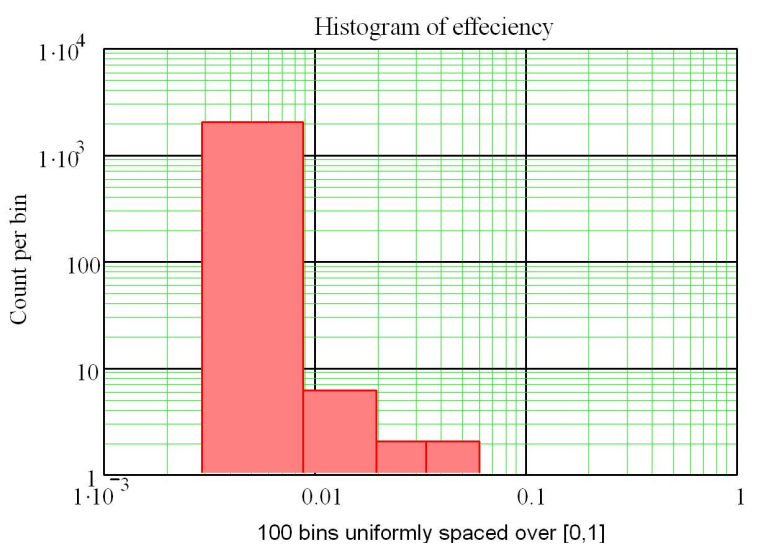

Fig. 5. TEM Horn efficiency histogram for $m=6$

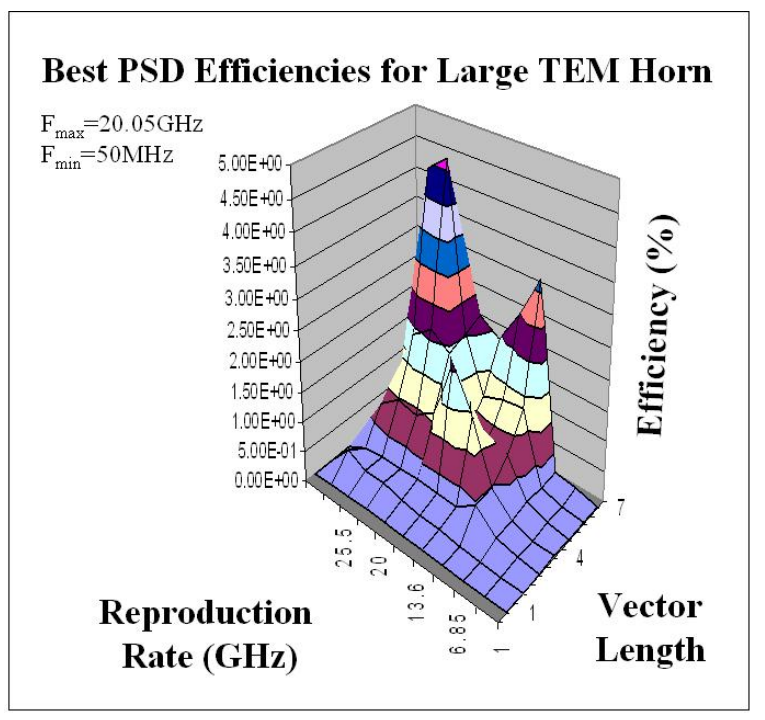

Fig. 6. TEM Horn 3D efficiency summary $(m=1,2 \ldots, 7)$

effort illustrates the complexity of the design space, and the seemingly limitless variety of hardware representations $H_{\text {eq }}(f)$ that can affect the optimal pulse generator design. The efficiencies of the sample designs given here are low, but there is room for significant improvement by decreasing the reproduction time $T_{s}$, increasing the number of quantization levels $|\mathcal{Q}|$, increasing the time support of the pulse waveform $p(t)$, inserting high-pass filters in the signal path to assist in staying below the mask for $f<3.1 \mathrm{GHz}$, etc.

\section{ACKNOWLEDGMENT}

This work was supported in part by a MURI Project under Contract DAAD19-01-1-0477 from the US Army Research Laboratory. The authors would like to thank the Raytheon Corporation for research support, specifically, Raytheon's CEO William H. Swanson, Kevin Hayata, David Derby, Dale R. Feikema and Mark Bianco. We also thank David Pozar, Won Namgoong, Shusaku Shimada and Clark Robertson for helpful discussions. And finally, we thank Jeff Yang, Robert Weaver, Majid Nemati, Robert Wilson and Meng-Hsuan Chung of the UltRa Lab for antenna support and finally Jordan Melzer for software support.

\section{REFERENCES}

[1] Federal Communications Commission, "Revision of Part 15 of the Commision's rules Regarding Ultra-Wideband Transmission Systems: First report and order, " Technical Report FCC 02-48 (adopted February 14, 2002; released April 22, 2002).

[2] R. A. Scholtz, "How Do you Define Bandwidth?" Proc. of the International Telemetering Conference, Los Angeles, California, October 1972.

[3] M.K. Simon, S. M. Hinedi and W. C. Lindsey, Digital Communication Techniques: Signal Design and Detection, Chapter 2, Prentice Hall PTR, 1st edition, Sept 19, 1994.

[4] D. Slepian, H. J. Laundau, and H. O. Pollack, Prolate Spheroidal Wave Functions, Fourier Analysis, and Uncertainty Principle I and II, The Bell System Technical Journal, vol 40, no 1, pp. 43-84, 1961.

[5] D. Slepian, Prolate spheroidal wave functions, Fourier analysis, and uncertainty - V: The discrete case. The Bell System Technical Journal, vol 57, no 5, May 1978 .

[6] A. Papoulis, Digital filtering and Prolate Functions, IEEE Transactions on Circuit Theory, vol CT-19, no 6, Nov. 1972.

[7] A. Papoulis, Signal Analysis, McGraw-Hill, Inc, 1977.

[8] T. Verma, S. Bilbao and T. Meng, The Digital Prolate Spheroidal Window, Proceedings IEEE ICASSP, pp 1351-1354, Atlanta Ga, May 1996.

[9] B. Parr, B. Cho, K. Wallace and Z. Ding, A Novel Ultra-Wideband Pulse Design Algorith, IEEE Communications Letters, vol. 7, No. 5, May 2003.

[10] J. Barth and J. Richner, "Distortion of Fast Pulses by Non-TEM Effects in Coaxial Cables," Ultra-Wideband, Short Pulse Electromagnetics 2, Edited by L. Carin and L. B. Felsen, Plenum Press, New York, 1995.

[11] A.O. Boryssenko, D.H. Schaubert, "Efficient and Practical Pulses for Dipole Antenna UWB Link," Proceedings of IEEE Ant. Propagat. Symp., June 2004.

[12] A. O. Boryssenko and D. H. Schaubert, "Time-Domain IntegralEquation-Based Solver for Transient and Broadband Problems in Electromagnetics," The AMEREM 2002 Symposium, Annapolis MD, June 2002.

[13] D. M. Pozar, "Waveform Optimizations for Ultra-Wideband Radio Systems," IEEE Trans. Antennas and Propagation, September 2003.

[14] R. Clark Robertson and Micheal A. Morgan, "Ultra-Wideband Impulse Receiving Antenna Design and Evaluation," Ultra-Wideband, Short-Pulse Electromagnetics 2, Edited by Lawrence Carin and Leopold B. Felsen, pp 179-186, Plenum Press, New York and London, 1995.

[15] R. Clark Robertson and Micheal A. Morgan, "Optimized TEM Horn Impulse Receiving Antenna," Ultra-Wideband, Short-Pulse Electromagnetics 3, Edited by Baum et al., pp 121-128, Plenum Press, New York, 1997.

[16] R. Clark Robertson and Micheal A. Morgan, Ultra-wideband Impulse Antenna Study and Prototype Design, Naval Postgraduate School Technical Report, NPSEC-93010, March 1993.

[17] J. A. Andrews, UWB signal Sources, Antennas and Propagation, Application Note AN-14a, Copyright August, 2003. 